\title{
The existence of solutions of $q$-difference-differential equations
}

\author{
Xin-Li Wang ${ }^{1}$, Hua Wang ${ }^{2}$ and Hong-Yan Xu 2* $^{*}$
}

${ }^{*}$ Correspondence: xuhongyan@jci.edu.cn ${ }^{2}$ Department of Informatics and Engineering, Jingdezhen Ceramic Institute,

Jingdezhen 333403, Jiangxi, People's Republic of China

Full list of author information is available at the end of the article

\begin{abstract}
By using the Nevanlinna theory of value distribution, we investigate the existence of solutions of some types of non-linear q-difference differential equations. In particular, we generalize the Rellich-Wittich-type theorem and Malmquist-type theorem about differential equations to the case of $q$-difference differential equations (system).
\end{abstract}

Keywords: Transcendental, $q$-Difference differential equation, Solution, Zero order

Mathematics Subject Classification: 39A 50, 30D 35

\section{Background}

In this paper, we shall assume that readers are familiar with the basic theorems and the standard notations of the Nevanlinna value distribution theory of meromorphic functions such as $m(r, f), N(r, f), T(r, f), \ldots$, (see Hayman 1964; Yang 1993; Yi and Yang 1995). For a meromorphic function $f, S(r, f)$ denotes any quantity satisfying $S(r, f)=o(T(r, f))$ for all $r$ outside a possible exceptional set of finite logarithmic measure, $\mathbb{S}(f)$ denotes the family of all meromorphic function $a(z)$ such that $T(r, a)=S(r, f)=o(T(r, f))$, where $r \rightarrow \infty$ outside of a possible exceptional set of finite logarithmic measure. In addition, we denote by $S_{1}(r, f)$ any quantity satisfying $S_{1}(r, f)=o(T(r, f))$ for all $r$ on a set $F$ of logarithmic density 1 , the logarithmic density of a set $F$ is defined by

$$
\limsup _{r \rightarrow \infty} \frac{1}{\log r} \int_{[1, r] \cap F} \frac{1}{t} d t
$$

Throughout this paper, the set $F$ of logarithmic density can be not necessarily the same at each occurrence.

Complex differential equations have attracted many mathematicians, and there are many results about the existence or growth of solutions of differential equations (see $\mathrm{He}$ 1981; Laine 1993, 1971; Liao 2015; Tu et al. 2013). In recent, with the development of Nevanlinna theory in complex difference equations (see Barnett et al. 2007; Chiang and Feng 2008; Gundersen et al. 2002; Halburd and Korhonen 2006a, b), there has been an increasing interest in studying difference equations, difference product and $q$-difference in the complex plane $\mathbb{C}$, a number of papers (including Chen 2010; Gan 2015; Halburd and Korhonen 2007; Heittokangas et al. 2001; Laine and Yang 2007; Qi and Yang 2015; 
Zheng and Chen 2010; Zhang and Korhonen 2010) have focused on the existence and growth of solutions of difference equation.

The following two results had been proved by F. Rellich and H. Wittich, respectively.

Theorem 1 (see He 1981, Rellich). Let the differential equation be the following form

$$
w^{\prime}(z)=f(w),
$$

If $f(w)$ is transcendental meromorphic function of $w$, then Eq. (1) has no non-constant entire solution.

Wittich (1955) studied the more general differential equation than Eq. (1) and obtained the following result.

Theorem 2 (see Wittich 1955). Let

$$
\Phi(z, w)=\sum a_{(i)}(z) w^{i_{0}}\left(w^{\prime}\right)^{i_{1}} \cdots\left(w^{(n)}\right)^{i_{n}}
$$

be differential polynomial, with coefficients $a_{(i)}(z)$ are polynomial of $z$. If the right-hand side of the differential equation

$$
\Phi(z, w)=f(w),
$$

$f(w)$ is the transcendental meromorphic function of $w$, then the Eq. (2) has no non-constant entire solution.

In the 1980s, Yanagihara and Shimomura extended the above type theorem to the case of difference equations (see Yanagihara 1980, 1983; Shimomura 1981), and obtained the following two results

Theorem 3 (see Shimomura 1981). For any non-constant polynomial $P(w)$, the difference equation

$$
w(z+1)=P(w(z))
$$

has a non-trivial entire solution.

Theorem 4 (see Yanagihara 1980). For any non-constant rational function $R(w)$, the difference equation

$$
w(z+1)=R(w(z))
$$

has a non-trivial meromorphic solution in the complex plane.

\section{Conclusions and our main results}

In the present paper, we mainly study the above Rellich-Wittich-type theorem of $q$-difference differential equation (system).

Definition 5 We call the equation a q-difference differential equation (system) if a equation (system) contains the $q$-difference term $f(q z)$ and differential term $f^{\prime}(z)$ of one function $f(z)$ at the same time.

We consider the system of $q$-difference differential equation of the form 


$$
\left\{\begin{array}{l}
\Omega_{1}\left(z, w_{1}\right):=\sum_{J_{1}} a_{J_{1}}(z) \prod_{j=1}^{n_{1}}\left(w_{1}^{(j)}\left(q_{j} z\right)\right)^{i_{j}}=P_{s}\left[f\left(w_{2}\right)\right], \\
\Omega_{2}\left(z, w_{2}\right):=\sum_{J_{2}} b_{J_{2}}(z) \prod_{j=1}^{n_{2}}\left(w_{2}^{(j)}\left(q_{j} z\right)\right)^{i_{j}}=P_{t}\left[f\left(w_{1}\right)\right],
\end{array}\right.
$$

where $a_{J_{1}}(z), b_{J_{2}}(z)$ are polynomials of $z$ and $q \in \mathbb{C} \backslash\{0\}, P_{m}[f]$ is a polynomial of $f$ of degree $m$,

$$
P_{m}[f]=d_{m}(z) f^{m}+d_{m-1}(z) f^{m-1}+\cdots+d_{0}(z),
$$

and $d_{m}(z), \ldots, d_{0}(z)$ are polynomials of $z$, and obtain the following results.

Theorem 6 For system (3), if $s \geq 1, t \geq 1$ and $f$ is a transcendental meromorphic function, then the system (3) has no non-constant transcendental entire solutions $\left(w_{1}, w_{2}\right)$ with zero order.

Remark 7 Under the assumptions of Theorem 6, the system of $q$-difference differential equation

$$
\left\{\begin{array}{l}
\sum_{J_{1}} a_{J_{1}}(z) \prod_{j=1}^{n_{1}}\left(w_{1}^{(j)}\left(q_{j} z\right)\right)^{i_{j}}=\frac{P_{s_{2}}\left[f\left(w_{2}\right)\right]}{Q_{t_{2}}\left[f\left(w_{2}\right)\right]}, \\
\sum_{J_{2}} b_{J_{2}}(z) \prod_{j=1}^{n_{2}}\left(w_{2}^{(j)}\left(q_{j} z\right)\right)^{i_{j}}=\frac{P_{s_{1}}\left[f\left(w_{1}\right)\right]}{Q_{t_{1}}\left[f\left(w_{1}\right)\right]},
\end{array}\right.
$$

has no non-constant transcendental entire solutions $\left(w_{1}, w_{2}\right)$ with zero order, where $s_{1}, s_{2} \geq 1$ and $P_{s_{i}}[f]$ and $Q_{t_{i}}[f]$ are irreducible polynomials in $f$.

If $s=t$ and $w_{1}=w_{2}$, we can get the following theorem easily

Theorem 8 Let

$$
\Omega(z, w):=\sum_{J} a_{J}(z) \prod_{j=1}^{n}\left(w^{(j)}\left(q_{j} z\right)^{i_{j}}\right)=P_{s}[f(w)],
$$

if $s \geq 1$ and $f$ is a transcendental meromorphic function, then the system (4) has no nonconstant transcendental entire solution with zero order.

From Remark 7, we have

Remark 9 Let $s \geq 1$ and $f$ be a transcendental meromorphic function, then the equation

$$
\sum_{J} a_{J}(z) \prod_{j=1}^{n}\left(w^{(j)}\left(q_{j} z\right)^{i_{j}}\right)=\frac{P_{s}[f(w)]}{Q_{t}[f(w)]},
$$


has no non-constant transcendental entire solution with zero order, where $P_{s}[f]$ and $Q_{t}[f]$ are irreducible polynomials in $f$.

As we know, it is very interest problem about the Malmquist theorem of differential equations, Laine (1993) gave the following results

Theorem 9 (see Laine 1993). Let

$$
\left(w^{\prime}(z)\right)^{n}=R(z, w),
$$

where $R(z, w)$ is defined as

$$
R(z, w)=\frac{\sum_{i=0}^{k} a_{i}(z)}{\sum_{j=0}^{l} b_{j}(z)} .
$$

If Eq. (5) has transcendental meromorphic solution, then there will be $l=0$ and $k \leq 2 n$.

Theorem 10 (see Laine 1993). Let

$$
\sum a_{(i)}(z) w^{i_{0}}\left(w^{\prime}\right)^{i_{1}} \cdots\left(w^{(n)}\right)^{i_{n}}=R(z, w),
$$

where $R(z, w)$ is defined as in Theorem 9. If Eq. (6) has transcendental meromorphic solution, then there will be $l=0$ and $k \leq \min \{\Delta, \lambda+\mu(1-\Theta(\infty))\}$, where

$$
\Delta=\max \left\{\sum_{\alpha=0}^{n}(1+\alpha) i_{\alpha}\right\}, \lambda=\max \left\{\sum_{\alpha=0}^{n} i_{\alpha}\right\},
$$

and

$$
\mu=\max \left\{\sum_{\alpha=0}^{n} \alpha i_{\alpha}\right\}, \quad \Theta(\infty)=1-\limsup _{r \rightarrow \infty} \frac{\bar{N}(r, w)}{T(r, w)} .
$$

Recently, there were a number of papers focused on the Malmquist-type theorem of the complex difference equations. Ablowitz et al. (2000) proved some results on the classical Malmquist-type theorem of the complex difference equations by applying Nevanlinna theory. Besides, Gao, Xu and Li also studied some systems of complex difference equation and obtained some more precise results related to Malmquist-type theorem (see Gao 2012a, b, c; Li and Gao 2015; Xu et al. 2013, 2015; Xu and Xuan 2015). In this paper, we mainly study the $q$-difference differential equation about the Maimquist-type theorem, and obtain the following theorem.

Theorem 11 Let

$$
\left(w^{\prime}(q z)\right)^{n}=R(z, w),
$$

where $R(z, w)$ is defined as

$$
R(z, w)=\frac{P(z, w)}{Q(z, w)}=\frac{\sum_{i=0}^{k} a_{i}(z) w^{i}}{\sum_{j=0}^{l} b_{j}(z) w^{j}},
$$


$P(z, w)$ and $Q(z, w)$ are irreducible polynomials in $w$, coefficients $a_{i}(z), b_{j}(z)$ are rational functions of $z$. If Eq. (7) exists transcendental meromorphic solutions with zero order, then we also think that $l=0$ and $k \leq 2 n$.

Similar to the proof of Theorem 11, we can get the following corollary easily.

Corollary 12 Let

$$
\sum a_{(i)}(z) w^{i_{0}}\left(w^{\prime}\right)^{i_{1}}\left(q_{1} z\right) \cdots\left(w^{(n)}\left(q_{n} z\right)\right)^{i_{n}}=R(z, w),
$$

where $R(z, w)$ is defined as in Theorem 11. If Eq. (8) has transcendental meromorphic solution of zero order, then there will be $l=0$ and $k \leq \min \{\Delta, \lambda+\mu(1-\Theta(\infty))\}$, where $\Delta, \lambda$ and $\mu$ are stated as in Theorem 10.

\section{Some Lemmas}

Lemma 13 (Valiron-Mohon'ko, Laine 1993). Let $f(z)$ be a meromorphic function. Then for all irreducible rational functions in $f$,

$$
R(z, f(z))=\frac{\sum_{i=0}^{m} a_{i}(z) f(z)^{i}}{\sum_{j=0}^{n} b_{j}(z) f(z)^{j}},
$$

with meromorphic coefficients $a_{i}(z), b_{j}(z)$, the characteristic function of $R(z, f(z))$ satisfies

$$
T(r, R(z, f(z)))=d T(r, f)+O(\Psi(r)),
$$

where $d=\max \{m, n\}$ and $\Psi(r)=\max _{i, j}\left\{T\left(r, a_{i}\right), T\left(r, b_{j}\right)\right\}$.

Lemma 14 (Zhang and Korhonen 2010, Theorem 1 and Theorem 3) Let $f(z)$ be a transcendental meromorphic function of zero order and $q$ be a nonzero complex constant. Then

$$
T(r, f(q z))=(1+o(1)) T(r, f(z))
$$

and

$$
N(r, f(q z))=(1+o(1)) N(r, f(z))
$$

on a set of logarithmic density 1.

Lemma 15 (see Barnett et al. 2007). Let $f(z)$ be a nonconstant zero-order meromorphic function and $q \in \mathbb{C} \backslash\{0\}$. Then

$$
m\left(r, \frac{f(q z)}{f(z)}\right)=S(r, f)
$$

on a set of logarithmic density 1 for all $r$ outside a possible exceptional set of logarithmic density 0 .

Lemma 16 (see Yi and Yang 1995, p. 37 or Yang 1993). Let $f(z)$ be a nonconstant meromorphic function in the complex plane and l be a positive integer. Then 


$$
N\left(r, f^{(l)}\right)=N(r, f)+l \bar{N}(r, f), \quad T\left(r, f^{(l)}\right) \leq T(r, f)+l \bar{N}(r, f)+S(r, f) .
$$

Lemma 17 Let $q \in \mathbb{C} \backslash\{0\}$ and $f(z)$ be a nonconstant meromorphic function with zero order. Then for any positive finite integer $k$, we have

$$
m\left(r, \frac{f^{(k)}(q z)}{f(z)}\right)=S_{1}(r, f),
$$

and

$$
m\left(r, f^{(k)}(q z)\right) \leq m(r, f)+S_{1}(r, f) .
$$

Proof It follows from Lemma 15 that

$$
m\left(r, \frac{f^{(k)}(q z)}{f(z)}\right) \leq m\left(r, \frac{f^{(k)}(q z)}{f(q z)}\right)+m\left(r, \frac{f(q z)}{f(z)}\right)=S_{1}(r, f) .
$$

Moreover, we have

$$
m\left(r, f^{(k)}(q z)\right)=m\left(r, \frac{f^{(k)}(q z)}{f(z)} f(z)\right) \leq m(r, f)+S_{1}(r, f) .
$$

This completes the proof of Lemma 17.

\section{The Proof of Theorem 6}

Suppose that $w_{i}(i=1,2)$ be non-constant entire functions solutions of system (3) with zero order. Suppose $i=1$, let $E_{1}=\left\{z:\left|w_{1}(z)\right|>1\right\}$ and $E_{2}=\left\{z:\left|w_{1}(z)\right| \leq 1\right\}$, then we have

$$
\begin{aligned}
\left|\Omega_{1}\left(z, w_{1}\right)\right| & =\left|\sum_{J_{1}} a_{J_{1}}(z)\left(w_{1}(z)\right)^{\lambda_{i}}\left(\frac{w_{1}^{\prime}\left(q_{1} z\right)}{w_{1}(z)}\right)^{i_{1}} \cdots\left(\frac{w_{1}^{\prime}\left(q_{n_{1}} z\right)}{w_{1}(z)}\right)^{i_{n_{1}}}\right| \\
& \leq \begin{cases}\left|w_{1}(z)\right|^{\lambda} \sum_{J_{1}}\left|a_{J_{1}}(z)\right|\left|\frac{w_{1}^{\prime}\left(q_{1} z\right)}{w_{1}(z)}\right|^{i_{1}} \cdots\left|\frac{w_{1}^{\prime}\left(q_{n_{1}} z\right)}{w_{1}(z)}\right|^{i_{n_{1}}}, & \text { if } z \in E_{1}, \\
\sum_{J_{1}}\left|a_{J_{1}}(z)\right|\left|\frac{w_{1}^{\prime}\left(q_{1} z\right)}{w_{1}(z)}\right|^{i_{1}} \ldots\left|\frac{w_{1}^{\prime}\left(q_{n_{1}} z\right)}{w_{1}(z)}\right|^{i_{n_{1}},}, & \text { if } z \in E_{2},\end{cases}
\end{aligned}
$$

where $\lambda=\max \left\{\lambda_{i}\right\}, \lambda_{i}=i_{1}+\cdots+i_{n_{1}}$. It follows from Lemma 15 and 17 that

$$
m\left(r, \Omega_{1}\left(z, w_{1}\right)\right)=\frac{1}{2 \pi}\left(\int_{E_{1}}+\int_{E_{2}}\right) \log ^{+}\left|\Omega_{1}\left(z, w_{1}\right)\right| d \theta \leq \lambda m\left(r, w_{1}\right)+S_{1}\left(r, w_{1}\right) .
$$

And since $w_{1}(z)$ is a non-constant entire function, we have $N\left(r, w_{1}\right)=0$. Thus, we have $N\left(r, \Omega_{1}\left(z, w_{1}\right)=0\right.$ and

$$
T\left(r, \Omega_{1}\right)=m\left(r, \Omega_{1}\right) \leq \lambda m\left(r, w_{1}\right)+S_{1}\left(r, w_{1}\right) .
$$

Similarly, we have

$$
T\left(r, \Omega_{2}\right)=m\left(r, \Omega_{2}\right) \leq \eta m\left(r, w_{2}\right)+S_{1}\left(r, w_{2}\right),
$$

where $\eta=\max \left\{\eta_{i}\right\}, \eta_{i}=i_{1}+\cdots+i_{n_{2}}$. 
Since $P_{s}\left[f\left(w_{2}\right)\right]$ is a polynomial of $f\left(w_{2}\right)$, we can take a complex constant $\alpha$ such that

$$
P_{s}\left[f\left(w_{2}\right)\right]-\alpha=\left[f\left(w_{2}\right)-\beta_{1}\right] \cdots\left[f\left(w_{2}\right)-\beta_{s}\right],
$$

where $\beta_{1}, \ldots, \beta_{s}$ are complex constants, and there at least exists a constant $\beta \in\left\{\beta_{1}, \ldots\right.$, $\left.\beta_{s}\right\}$ which is not Picard exceptional value of $f\left(w_{2}\right)$. Let $\left\{\xi_{j}, j=1,2, \ldots, p_{2}\right\}$ be the zeros of $f\left(w_{2}\right)-\beta$, where $p_{2}$ is any positive integer with $p_{2} \geq 1$. Then it follows

$$
\sum_{j=1}^{p_{2}} N\left(r, \frac{1}{w_{2}-\xi_{j}}\right) \leq N\left(r, \frac{1}{f\left(w_{2}\right)-\beta}\right) \leq N\left(r, \frac{1}{P_{s}\left[f\left(w_{2}\right)\right]-\alpha}\right) .
$$

Thus, by using the second main theorem and (10), (11), we can get that

$$
\begin{aligned}
\left(p_{2}-2\right) T\left(r, w_{2}\right) & \leq \sum_{j=1}^{p_{2}} N\left(r, \frac{1}{w_{2}-\xi_{j}}\right)+S\left(r, w_{2}\right) \\
& \leq N\left(r, \frac{1}{P_{s}\left[f\left(w_{2}\right)\right]-\alpha}\right)+S\left(r, w_{2}\right) \\
& \leq T\left(r, P_{s}\left[f\left(w_{2}\right)\right]\right)+S\left(r, w_{2}\right) \\
& \leq T\left(r, \Omega_{1}\left(z, w_{1}\right)\right)+S\left(r, w_{2}\right) \\
& \leq \lambda T\left(r, w_{1}\right)+S_{1}\left(r, w_{1}\right)+S_{1}\left(r, w_{2}\right) .
\end{aligned}
$$

Similarly, there exists any positive integer $p_{1}(\geq 1)$ such that

$$
\left(p_{1}-2\right) T\left(r, w_{1}\right) \leq \eta T\left(r, w_{2}\right)+S_{1}\left(r, w_{1}\right)+S_{1}\left(r, w_{2}\right) .
$$

It follows from (12) and (13) that

$$
\left[\left(p_{1}-2\right)\left(p_{2}-2\right)-\lambda \eta\right] T\left(r, w_{i}\right) \leq S_{1}\left(r, w_{1}\right)+S_{1}\left(r, w_{2}\right),
$$

Since $w_{i}(i=1,2)$ are transcendental and $p_{1}, p_{2}$ are arbitrary, we can get a contradiction with (4). Hence, we complete the proof of Theorem 6.

\section{The Proof of Theorem 11}

We firstly choose a constant $a \in \mathbb{C}$ such that $P(z, a) \neq 0$ and $Q(z, a) \neq 0$, then (7) can be rewritten as

$$
\left(w^{\prime}(q z)\right)^{n}=\frac{P(z, a)+A_{1}(z)(w-a)+\cdots+A_{k}(z)(w-a)^{k}}{Q(z, a)+B_{1}(z)(w-a)+\cdots+B_{l}(z)(w-a)^{l}},
$$

where $A_{1}(z), \ldots, A_{k}(z), B_{1}(z), \ldots, B_{l}(z)$ are all rational functions. Let $\varphi(z)=\frac{1}{w(z)-a}$, that is, $w(z)=\frac{1}{\varphi(z)}+a$ and

$$
\left(w^{\prime}(q z)\right)^{n}=(-1)^{n} \varphi(q z)^{-2 n}\left(\varphi^{\prime}(q z)\right)^{n} .
$$


Hence, it follows from (15) and (16) that

$$
\begin{aligned}
\left(\varphi^{\prime}(q z)\right)^{n} & =(-1)^{-n} \varphi(q z)^{2 n}\left(w^{\prime}(q z)\right)^{n} \\
& =(-1)^{-n} \varphi(q z)^{2 n} \frac{P(z, a)+A_{1}(z) \varphi(z)^{-1}+\cdots+A_{k}(z) \varphi(z)^{-k}}{Q(z, a)+B_{1}(z) \varphi(z)^{-1}+\cdots+B_{l}(z) \varphi(z)^{-l}} \\
& =: \varphi(q z)^{2 n} \varphi(z)^{l-k} \frac{\sum_{i=0}^{k} \widetilde{a}_{i}(z) \varphi(z)^{i}}{\sum_{i=0}^{l} \widetilde{b}_{i}(z) \varphi(z)^{i}} \\
& =\frac{\widetilde{P}(z, \varphi(z))}{\widetilde{Q}(z, \varphi(z))}=\widetilde{R}(z, \varphi(z)),
\end{aligned}
$$

where $\tilde{a}_{i}(z)=(-1)^{-n} P(z, a) \neq 0, \tilde{b}_{i}(z)=Q(z, a) \neq 0$.

Suppose that $w(z)$ is a transcendental meromorphic solution of equation (7) with zero order, then $\varphi(z)=\frac{1}{w(z)-a}$ is also a transcendental meromorphic solution of Eq. (17). We will discuss two cases as follows.

If $2 n+l-k \leq 0$, then $\operatorname{deg}_{\varphi} \widetilde{P}(z, \varphi)=k$ and $\operatorname{deg}_{\varphi} \widetilde{Q}(z, \varphi)=l-(2 n+l-k)=k-2 n$. It follows by Lemma 13 that

$$
T(r, \widetilde{R}(z, \varphi(z))=k T(r, \varphi)+S(r, \varphi) .
$$

And by Lemmas 13-17, we have

$$
\begin{aligned}
T\left(r,\left(\varphi^{\prime}(q z)\right)^{n}\right) & =n T\left(r, \varphi^{\prime}(q z)\right) \\
& \leq n N(r, \varphi(q z))+n \bar{N}\left(r, \varphi^{\prime}(q z)\right)+n m\left(r, \varphi^{\prime}(q z)\right) \\
& \leq 2 n T(r, \varphi(q z))+S(r, \varphi) \\
& \leq 2 n T(r, \varphi)+S_{1}(r, \varphi) .
\end{aligned}
$$

Thus, it follows

$$
k T(r, \varphi) \leq 2 n T(r, \varphi)+S_{1}(r, \varphi),
$$

which implies $k \leq 2 n$. Since $2 n+l-k \leq 0$ and $l \geq 0$, then we have $l=0$.

If $2 n+l-k \geq 0$, then then $\operatorname{deg}_{\varphi} \widetilde{P}(z, \varphi)=2 n+l$ and $\operatorname{deg}_{\varphi} \widetilde{Q}(z, \varphi)=l$. It follows by Lemma 13 that

$$
T(r, \widetilde{R}(z, \varphi(z))=(2 n+l) T(r, \varphi)+S(r, \varphi) .
$$

Similar to the argument as in above, we can get $l=0$ and $k \leq 2 n$.

This completes the proof of Theorem 11 .

Authors' contributions

XLW, HW and HYX completed the main part of this article. All authors read and approved the final manuscript.

\section{Author details}

${ }^{1}$ College of Science, University of Shanghai for Science and Technology, Shanghai 200093, People's Republic of China.

${ }^{2}$ Department of Informatics and Engineering, Jingdezhen Ceramic Institute, Jingdezhen 333403, Jiangxi, People's

Republic of China.

\section{Acknowledgements}

The authors were supported by the NSF of China $(11561033,11301233)$, the Natural Science Foundation of Jiangxi Province in China (20151BAB201008), and the Foundation of Education Department of Jiangxi (GJJ150902) of China.

\section{Competing interests}

The authors declare that they have no competing interests.

Received: 12 November 2015 Accepted: 19 April 2016

Published online: 04 May 2016 
References

Ablowitz MJ, Halburd R, Herbst B (2000) On the extension of the Painlevé property to differenceequations. Nonlinearity 13(3):889-905

Barnett DC, Halburd RG, Korhonen RJ, Morgan W (2007) Nevanlinna theory for the q-difference operator and meromorphic solutions of 9 -difference equations. Proc R Soc Edinb Sect A Math 137:457-474

Chen ZX (2010) Value distribution of meromorphic solutions of certain difference Painlev equations. J Math Anal Appl 364:556-566

Chiang YM, Feng SJ (2008) On the Nevanlinna characteristic of $f(z+\eta)$ and difference equations in the complex plane. Ramanujan J 16:105-129

Gan HL (2015) The zeros and fixed points of difference of entire functions.J Jiangxi Norm Univ Nat Sci 39(5):519-522

Gao LY (2012a) On meromorphic solutions of a type of system of composite functional equations. Acta Math Sci 32B(2):800-806

Gao LY (2012b) Systems of complex difference equations of Malmquist type. Acta Math Sin 55:293-300

Gao LY (2012c) Estimates of $\mathrm{N}$-function and $\mathrm{m}$-function of meromorphic solutions of systems of complex difference equations. Acta Math Sci 32B(4):1495-1502

Gundersen GG, Heittokangas J, Laine I, Rieppo J, Yang DQ (2002) Meromorphic solutions of generalized Schröder equations. Aequ Math 63:110-135

Halburd RG, Korhonen RJ (2006a) Difference analogue of the lemma on the logarithmic derivative with applications to difference equations. J Math Anal Appl 314:477-487

Halburd RG, Korhonen RJ (2006b) Nevanlinna theory for the difference operator. Ann Acad Sci Fenn Math 31:463-478

Halburd RG, Korhonen RJ (2007) Finite order solutions and the discrete Painlevé equations. Proc Lond Math Soc 94:443-474 Hayman WK (1964) Meromorphic functions. The Clarendon Press, Oxford

He YZ (1981) On the algebroid function solutions of differential equations. Acta Math Sin 24:464-471

Heittokangas J, Korhonen R, Laine I, Rieppo J, Hohge K (2001) Complex difference equations of Malmquist type. Comput Methods Funct Theory 1:27-39

Laine I (1971) On the behaviour of the solutions of some first order differential equations. Ann Acad Sci Fenn Ser A I 497:1-26 Laine I (1993) Nevanlinna theory and complex differential equations. Walter de Gruyter, Berlin

Laine I, Yang CC (2007) Clunie theorems for difference and q-difference polynomials. J Lond Math Soc 76(2):556-566

Liao LW (2015) The new developments in the research of nonlinear complex differential equations. J Jiangxi Norm Univ Nat Sci 39(4):331-339

Li HC, Gao LY (2015) Meromorphic solutions of a type of system of complex differential-difference equations. Acta Math Sci 35B(1):195-206

Qi XG, Yang LZ (2015) Properties of meromorphic solutions of a-difference equations. Electron J Differ Eq 2015(59):1-9

Shimomura S (1981) Entire solutins of a polynomial difference equation. J Fac Sci Univ Tokyo Sect IA Math 28:253-266

Tu J, Huang HX, Xu HY, Chen CF (2013) The order and type of meromorphic functions and analytic functions in the unit disc. J Jiangxi Norm Univ Nat Sci 37(5):449-452

Wittich H (1955) Neuere Untersuchungen über eindeutige analytische Funktionen. Springer, Berlin

Xu HY, Liu BX, Tang KZ (2013) Some properties of meromorphic solutions of systems of complex q-shift difference equations. In: Abstract and applied analysis, vol 2013, Art. 680956

$\mathrm{Xu} \mathrm{HY}$, Wang JL, Wang H (2015) The existence of meromorphic solutions of some types of systems of complex functional equations. In: Discrete dynamics in nature and society, vol 2015, Art. 723025

Xu HY, Xuan ZX (2015) Growth of the solutions of some q-difference differential equations. Adv Differ Eq. 2015:172

Yanagihara N (1980) Meromorphic solutionas of some difference equations. Funkc Ekvacioj 23:309-326

Yanagihara N (1983) Meromorphic solutionas of some difference equations of the nth order. Arch Ration Mech Anal 91:19-192

Yang L (1993) Value distribution theory. Springer, Berlin

Yi HX, Yang CC (1995) Uniqueness theory of meromorphic functions, Kluwer Academic Publishers, Dordrecht, 2003; Chinese original. Science Press, Beijing

Zhang JL, Korhonen RJ (2010) On the Nevanlinna characteristic of $f(q z)$ and its applications. J Math Anal Appl 369:537-544

Zheng XM, Chen ZX (2010) Some properties of meromorphic solutions of q-difference equations. J Math Anal App $361: 472-480$

\section{Submit your manuscript to a SpringerOpen ${ }^{\circ}$ journal and benefit from:}

- Convenient online submission

- Rigorous peer review

- Immediate publication on acceptance

- Open access: articles freely available online

- High visibility within the field

- Retaining the copyright to your article

Submit your next manuscript at $\boldsymbol{\nabla}$ springeropen.com 